\title{
Simulated hemodynamics in human carotid bifurcation based on Doppler ultrasound data
}

\author{
Luísa C. Sousa', Catarina F. Castro ${ }^{1}$, Carlos C. António ${ }^{1}$, João M.R.S. Tavares², André M.F. Santos², \\ Rosa M. Santos ${ }^{3}$, Pedro Castro ${ }^{3}$, and Elsa Azevedo ${ }^{3}$
}

Special Issue on Neurosonology and Cerebral Hemodynamics

\begin{abstract}
Background: Atherosclerotic lesions commonly develop at arterial branch sites. Non-invasive carotid artery ultrasound is a well-established and effective method that allows real-time imaging and measurement of flow velocities. We aimed to develop a methodology for patient-specific computational 3D reconstruction and blood flow simulation based on ultrasound image data.
\end{abstract}

Methods: Subject-specific studies based on the acquisition of a set of longitudinal and sequential cross-sectional ultrasound images and Doppler velocity measurements at common carotid artery (CCA) bifurcation were performed at a university hospital. A developed simulation code of blood flow by the finite element method (FEM) that includes an adequate structured meshing of the common carotid artery bifurcation was used to investigate local flow biomechanics.

Results: Hemodynamic simulations of CCA bifurcations for six individuals were analyzed. Comparing pairs (Doppler, FEM) of velocity values, Lin's concordance correlation coefficient analysis demonstrated an almost perfect strength of agreement $\left(\rho_{c}=0.9911\right)$, in patients with different degrees of internal carotid artery (ICA) stenosis. Numerical simulations were able to capture areas of low wall shear stress correlated with stagnation zones.

Conclusion: Simulated hemodynamic parameters can reproduce the disturbed flow conditions at the bifurcation of CCA and proximal ICA, which play an important role in the development of local atherosclerotic plaques. This novel technology might help to understand the relationship between hemodynamic environment and carotid wall lesions, and have a future impact in carotid stenosis diagnosis and management.

Keywords: Doppler image-based analysis, Carotid bifurcation, Computational fluid dynamics, Wall shear stress.

'Institute of Mechanical Engineering (IDMEC-FEUP), Faculty of Engineering, University of Porto, Porto, Portugal

Institute of Mechanical Engineering and Industrial Management, Faculty of Engineering, University of Porto, Porto, Portugal

${ }^{3}$ Department of Neurology, Hospital São João, Faculty of Medicine,

University of Porto, Porto, Portugal
Citation: Sousa et al. Simulated hemodynamics in human carotid bifurcation based on Doppler ultrasound data. IJCNMH 2014; 1(Suppl. 1):S15

Received: 13 Sep 2013; Accepted: 20 Nov 2013; Published: 09 May 2014

\section{Correspondence: Luisa Costa Sousa}

Institute of Mechanical Engineering (IDMEC-FEUP), Faculty of Engineering, University of Porto

Rua Dr. Roberto Frias, s/n, 4200 - 465 Porto, Portugal

Email adress: Icsousa@fe.up.pt 


\section{Introduction}

It is widely accepted that, in large arteries, regions of disturbed flow like arterial bifurcations or curvatures correspond closely those locations where atherosclerosis develops. Atherosclerosis results from an accumulation of lipids and other materials in arterial walls, and can cause a focal luminal narrowing as well as loss of elasticity in the arteries. This disease often leads to heart attack and brain stroke, leading causes of death in the industrial world. Although the precise hemodynamic determinants of atherosclerotic disease are not yet completely understood, different studies have shown that atherosclerotic sites correlate strongly with regions of disturbed flow [1-3].

Doppler ultrasound imaging has been widely used in clinical practice to image blood vessels and to quantify arterial blood flow. B-mode ultrasound allows direct visualization of both vessel wall and lumen and, subsequently, detection of early atherosclerosis, beginning with intima-media thickening [4-6]. Such information is a prerequisite for accurate diagnosis and assessment of the severity of arterial disease, with all that this implies in terms of the treatment that individual patients receive.

Computational fluid dynamics (CFD) models have become very effective tools for predicting the flow field within the carotid bifurcation, and for understanding the relationship between local hemodynamics, and the initiation and progression of vascular wall pathologies. CFD, applied to realistic, three-dimensional arterial geometries derived from clinical imaging, provides an accurate assessment of flow patterns and shear stress in complex geometries $[7,8]$.

Recent work has demonstrated differences in carotid artery blood flow dynamics between individuals $[9,10]$ and the use of a suitable-scaled characteristic waveform is reasonable when subject-specific flow conditions are unavailable. Although for large-scale studies of common carotid artery (CCA) bifurcation hemodynamics the use of a typical flow waveform shape is likely sufficient, for a detailed patient study a subject-specific waveform collected in clinical practice, as in our study, yields a more accurate assessment of flow characteristics $[9,11,12]$.

The aim of the present investigation was to study of the image-based CFD of stenozed carotid bifurcations. Selecting patient specific stenozed carotid artery as diagnosed by ultrasound imaging, the complexity of the hemodynamic environment of five diseased CCA bifurcations is addressed and compared with a stenosis-free case.

\section{Methods}

Modeling a carotid bifurcation starting from medical images requires four serial steps: image acquisition, image-based definition of the carotid bifurcation model, computational simulation, and post-processing as described below.

In this study ultrasound data from six CCA bifurcations, referred in this study as P1 to P6, were analyzed. Patient ages ranged from 50 to 84 years. The present research received favorable opinion by the institutional Ethics Committee, and all subjects gave written informed consent.

\section{Acquisition of anatomical in vivo data}

The ultrasound imaging examinations were performed by an experienced certified sonographer, dedicated to neurovascular ultrasound at the Neurosonology Unit of the Department of Neurology of São João Hospital Centre, Oporto, Portugal. For each volunteer, a set of B-mode and pulsed-wave Doppler images of the CCA, its bifurcation, and proximal segments of the internal carotid artery (ICA) and the external carotid artery (ECA) was obtained. High-Resolution B-Mode Ultrasound scanner (Vivid e; GE, Milwaukee, WI, USA) was used to examine the extracranial carotid arteries. This system, equipped with a linear array transducer probe (GE 8L-RS) with pulsedwave Doppler and spectrum analysis capabilities, provides high-resolution ultrasonic images with a resolution of $614 \times 820$ pixels and 256 degrees of gray scale. Three-dimensional (3D) models of the lumen and wall boundaries were reconstructed from B-mode longitudinal images completed by B-mode cross-sectional images registered at the end of diastolic phase to control physiologic variations of vessel diameter along cardiac cycle. Carotid stenosis was measured according to the percentage of luminal diameter narrowing at the most stenotic point, according to ECST grading method [13]. An example of longitudinal and cross-sectional B-mode images for volunteer (P1) are presented in Figure 1.

Using pulsed wave mode, blood flow velocity spectral waveforms were obtained at several specific locations identified on B-mode imaging, from approximately $2 \mathrm{~cm}$ before CCA bifurcation, until post-bulbar ICA and ECA, including the bifurcation entrance (APEX). Angle correction was activated as appropriated, with angle of insonation $\leq 60^{\circ}$ [14]. Ultrasound images were stored to hard disc for later offline analysis.

\section{Geometrical 3D surface reconstruction}

We developed a semi-automatic methodology for reconstruction and structured meshing based on the images obtained with ultrasound. DICOM files were imported into a specific program developed in MATLAB (The Mathworks Inc. Natick, MA, USA). Then B-mode images were segmented to produce smooth lumen and plaque contours by using purpose-developed software [15-17], which automatically segments the lumen and bifurcation boundaries of the carotid artery based on the hypoechogenic characteristics of the lumen. Using this software each input image was initially processed with the application of an anisotropic diffusion filter for speckle noise removal, and morphologic operators were employed in the detection of the relevant ultrasound data regarding the artery. The information obtained was then used to define initial contours, corresponding to the lumen and to the bifurcation 

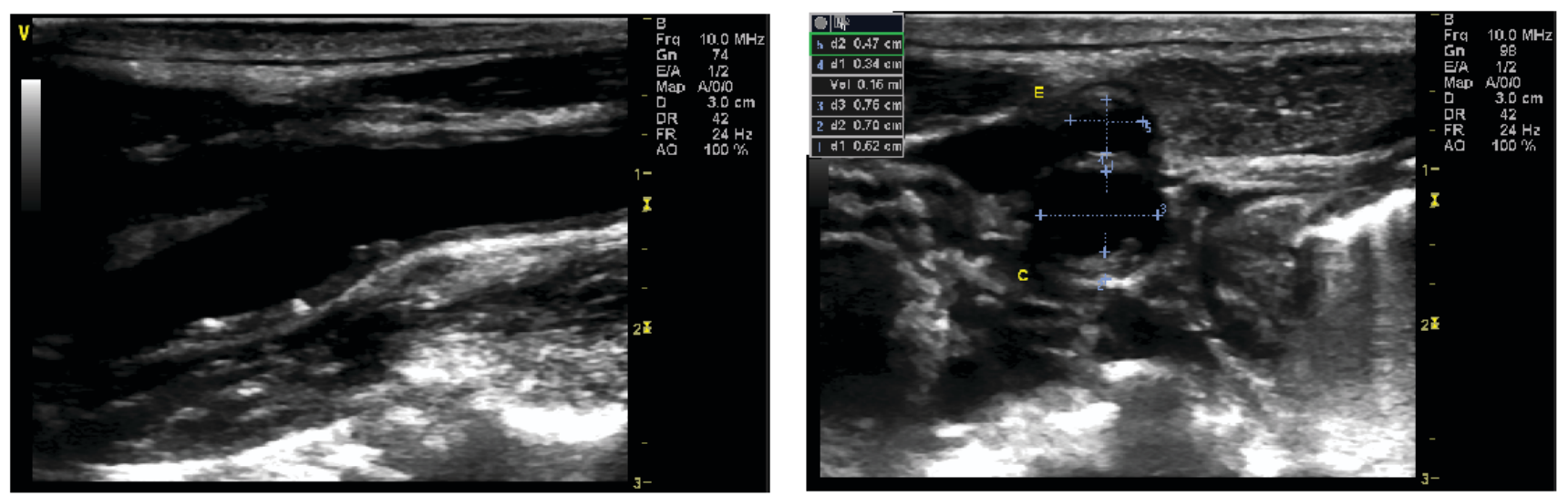

Figure 1. Ultrasound images of the volunteer P1 common carotid artery bifurcation region: longitudinal (left) and cross-sectional (right) images.

boundaries, for the application of the Chan-Vese level set segmentation model $[18,19]$.

Geometric lumen boundaries for CCA, ICA, and ECA were obtained importing the segmented 2D images into the modeling software FEMAP (FEMAP, Siemens PLM, USA \& Canada). Specific points of the lumen-intima and media-adventitia boundaries were identified in order to construct splines A, B, C and D for ICA, ECA and CCA boundary definition, as shown is Figure 2(a).

The centerlines of CCA, ECA and ICA were defined by creating a curve associated to equidistant points from splines A to B, A to C and D to B. Segmented transverse contours were then positioned and oriented in 3D space; each contour was then realigned so that its centroid coincided with the defined arteries centerlines. Figure 2(b) and Figure 2(c) show the specific 3D surface reconstruction for patient P1 defining the reconstructed bifurcation shape.

\section{Blood flow model}

Subject-specific 3D hemodynamics were determined using an in-house CFD code, which has been extensively validated [20, 21]. Eight-node hexahedral isoparametric element meshes constructed with software FEMAP were employed to discretize each bifurcation volume. Cylindrical flow extensions were extruded from the lower boundary in CCA (inlet) and the upper boundary in ICA and ECA (outlet) to facilitate the imposition of velocity boundary conditions as described below. Vessel walls were assumed to be rigid and blood was approximated by an isotropic, incompressible, homogeneous and Newtonian viscous fluid with a density of $1060 \mathrm{~kg} / \mathrm{m}^{3}$. The rheological behavior of blood was simulated considering a constant dynamic viscosity value of $0.0035 \mathrm{~kg} /(\mathrm{m} . \mathrm{s})$, a reasonable assumption for bulk flow metrics [22, 23]. Newtonian rheology is reasonable in the context of fluid precision and uncertainties related to boundary conditions [11,23-25].

A

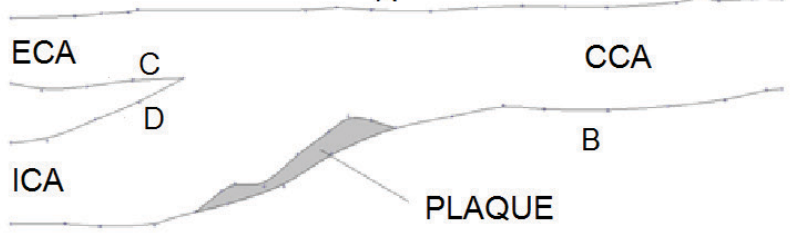

(a)

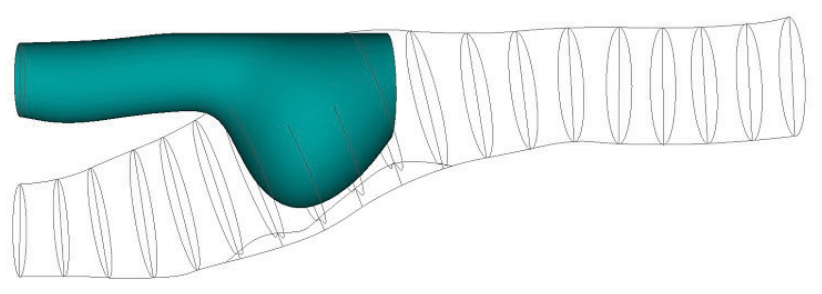

(b)

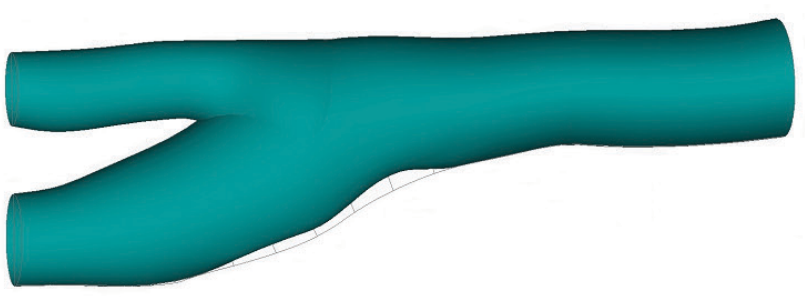

(c)

Figure 2. 3D model reconstruction: (a) definition of CCA, ICA and ECA lumen boundaries; (b) definition of cross-sections; (c) reconstructed surface of bifurcation P1. 
The volume-filling finite element meshes consisted of nearly 60000 quadratic hexahedral finite elements. The nonlinear system of equations derived from the discretization of the flow equations was solved using the upwinding method. The backward Euler implicit time integration scheme was implemented to obtain the solution at each time step of the time-dependent problem. Based upon mesh and time-step refinement studies [21], this mesh density and constant time-step equal to $2.5 \times 10^{-3}$ s were deemed sufficient for the purposes of characterizing velocities and wall shear stress (WSS) patterns.

For each bifurcation analysis, subject-specific velocity boundary conditions were prescribed at CCA inlet and ICA outlet using Womersley velocity profiles derived from pulsatile spectral waveforms obtained by pulsed Doppler and traction-free boundary condition was defined at ECA outlet [9, 26-28]. Figure 3 presents the volume hexahedral mesh (left) and the distal CCA velocity waveform (right) used for solving the hemodynamics of subject P1.

\section{Results}

Pulsatile hemodynamics were computed for six patients (ages 50 to 84 years; 4 males and 2 females) identified in Figure 4. The local degree of stenosis (ECST) was registered. For patient P3 no ICA plaque was observed and for the others, an ICA stenosis from 30 to $70 \%$ was registered. Peak systole velocities at ICA are presented in Figure 4. Comparing pairs (Doppler, FEM) of velocity values, Lin's concordance correlation coefficient analysis demonstrated an almost perfect strength of agreement $\left(\rho_{c}=0.9911\right)$ between ultrasound data and simulated FEM values.

Velocity field at peak systolic phase is shown in Figure 5 for patients P1 to P6. Bifurcation P3 is the non-stenotic carotid of the analyzed set and P4 to P6 present a higher degree of stenosis. For most patients a strongly skewed axial velocity in the proximal internal carotid artery due to enlarged bulb region was observed. For all patients a stagnation zone was detected near the outer bulb wall (opposite to the divider wall), as expected. At systole, patients P2 to P4 exhibit higher velocities at ECA as compared to the other patients, which present higher velocities at ICA.

At ECA, during systolic peak, high velocity gradients were detected for patients P3 and P4, due to the sharp unevenness of the vessel wall. At ICA, the highest velocity gradients were detected during peak systole for patients P1 and $\mathrm{P} 4$ to $\mathrm{P} 6$.

WSS contours near peak systole for all the volunteers (P1 to P6) are shown in Figure 6, exhibiting regions of low shear stress located on the lateral surface of proximal ICA. The main features expected from fluid dynamics, such as low WSS values in the bulb region of the ICA and high WSS in proximal ECA were successfully captured. Nevertheless, different WSS patterns were found for each individual, mainly due to the effect of patient-specific artery morphology variability. For the non-stenotic bifurcation P3, low WSS patches in CCA were contiguous with the carotid bulb low WSS region. For patients P1, P2, and P3, maximum values of WSS, equal to 32,35 , and $22 \mathrm{~Pa}$ respectively, were found at inner wall of ECA proximal to bifurcation. For the other patients presenting higher stenosis degree, maximum WSS were located at ICA within stenosis. The maximum value of WSS was $42 \mathrm{~Pa}$ and it was observed for patient P4.

\section{Discussion}

A noninvasive approach for simultaneously quantifying flow and WSS fields at CCA bifurcation was presented. Inter-individual variation in flow dynamics was analyzed considering six different models based on ultrasound morphologic and velocimetric acquisitions.
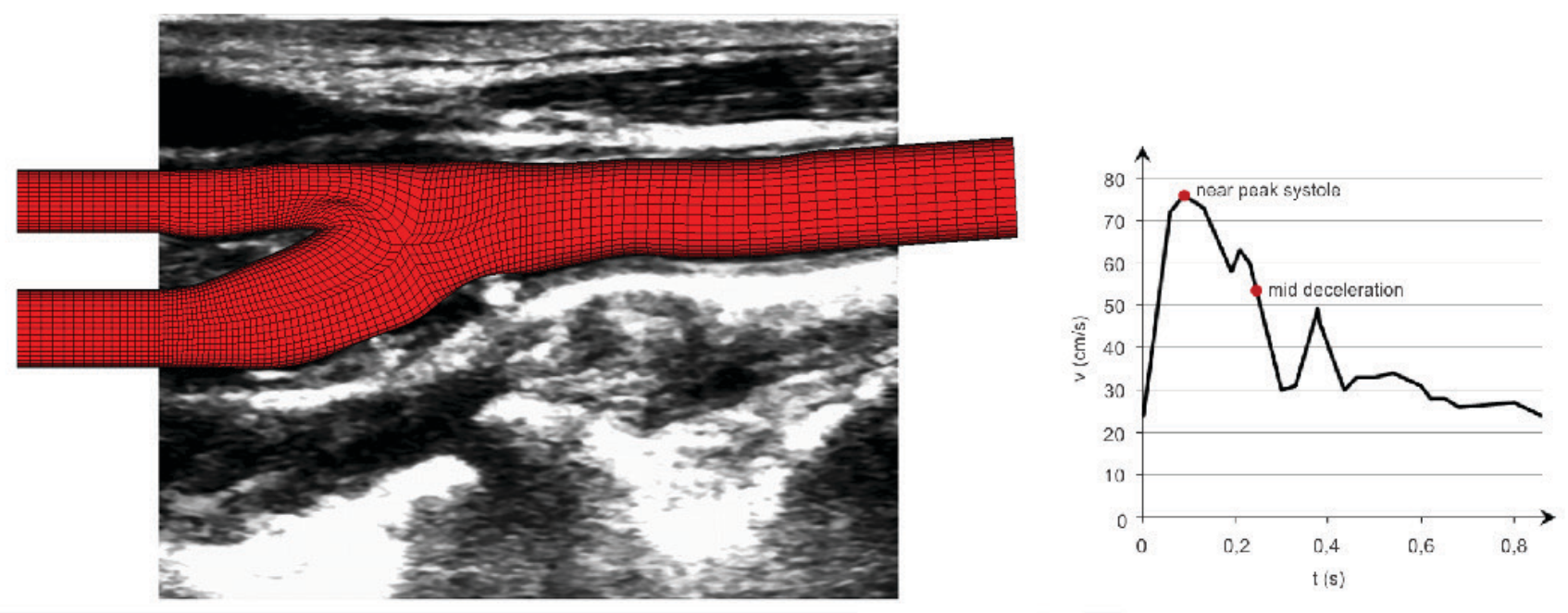

Figure 3. Volume discretization and distal CCA velocity waveform used for hemodynamics simulation of patient P1 bifurcation. 


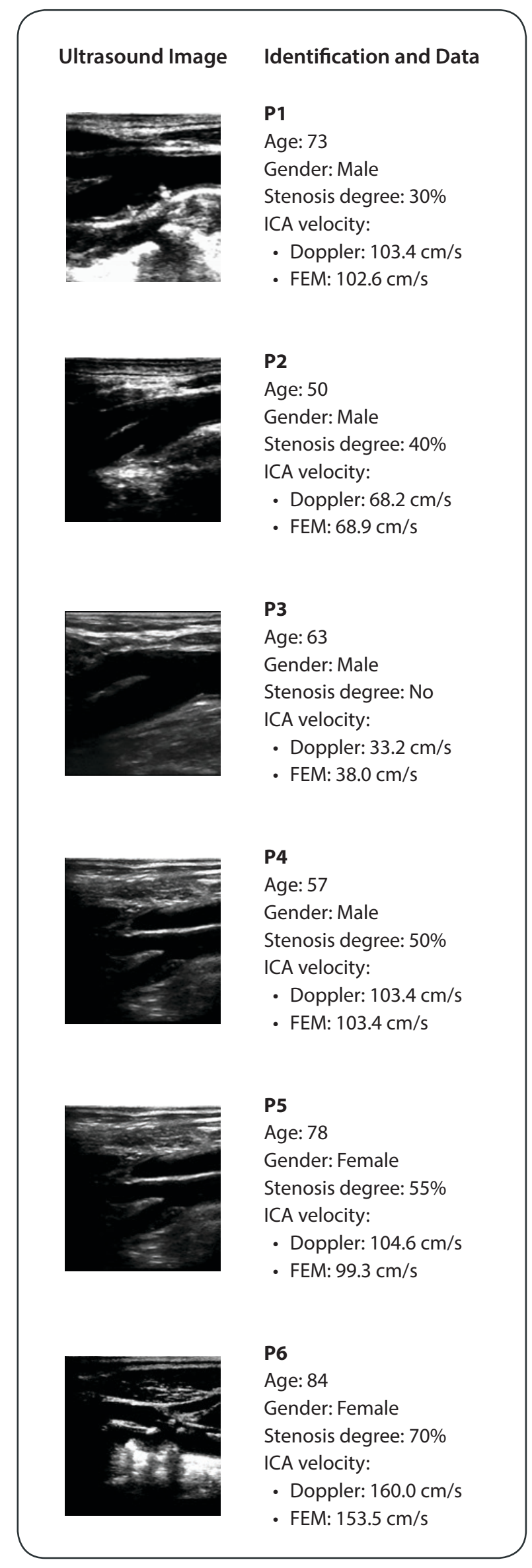

Figure 4. Longitudinal images and identification of patients P1 to P6.
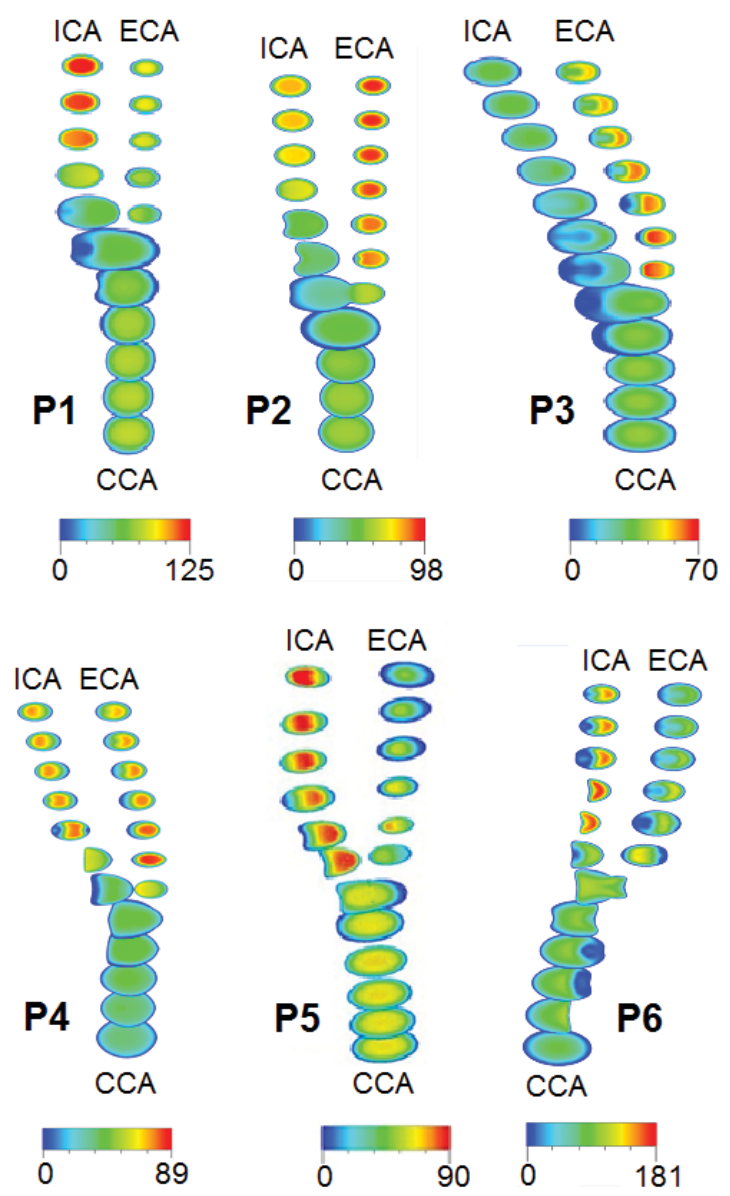

Figure 5. Velocity field $(\mathrm{cm} / \mathrm{s})$ at systolic peak for patients P1 to P6.

Each patient exhibits a different velocity pattern, associated with its morphology. When the bulb atherosclerotic plaques did not cause relevant stenosis, as in patients P1 and P2, maximum velocities at ICA appeared more distally, similarly to the P3 non-stenotic case, while for patients P4 to P6 maximum velocities were detected within stenosis, presenting high gradients.

Different WSS fields were found for the six volunteers as reported in Figure 6. For all patients with stenozed ICA, high WSS values were observed within the stenosis and low WSS values were detected in the non-stenotic or near normal ICA bulb region, and also in ICA distally to stenosis. For the non-stenotic bifurcation P3, low WSS patches in the common carotid artery (CCA) were contiguous with the carotid bulb low WSS region.

Since real artery morphology and flow velocities were employed, an obvious question arises if variations were attributable to morphology or flow velocity differences or both $[12,29,30]$. The analyzed bifurcations indicate that morphology, as the curvature of the in vivo models, may play the key role in determining the wall shear stress patterns. These findings could help to explain why some individuals develop more pronounced ICA stenosis than others, although vascular risk factors may be similar. 

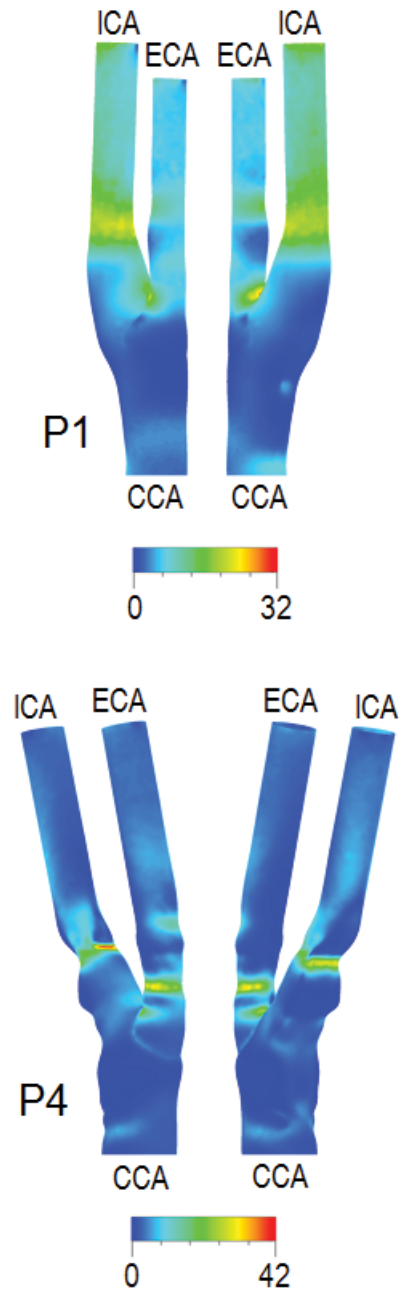
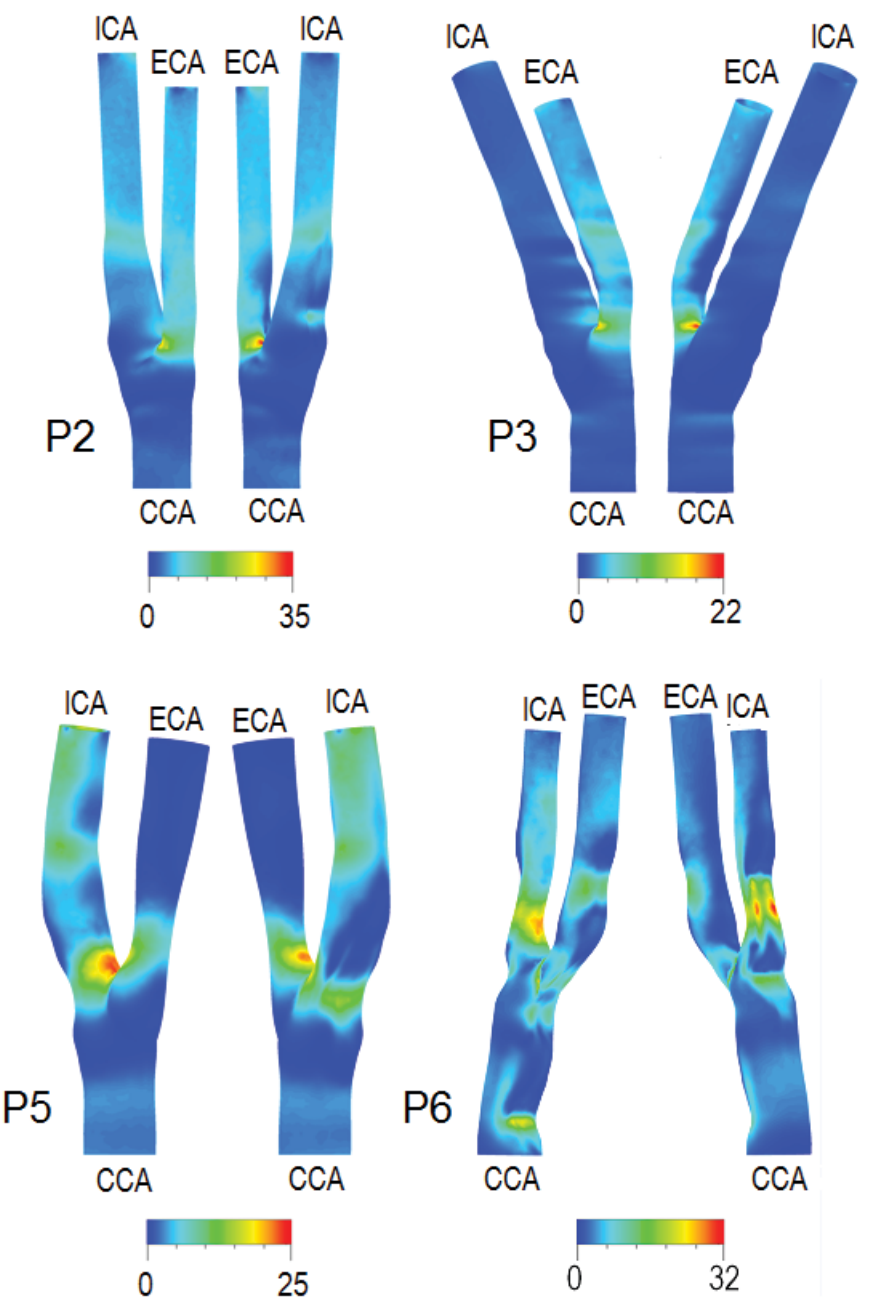

Figure 6. WSS contours (Pa) near peak systole for carotid bifurcations P1 to P6.

A full understanding of hemodynamic changes caused by the carotid bifurcation and stenosis is meaningful for clinical research. In this study we presented a noninvasive approach for simultaneously quantifying subject-specific flow patterns and wall shear stress distributions of human carotid bifurcation using a combination of ultrasound data and CFD modeling. Application of this novel approach to a normal volunteer and five subjects with atherosclerosis demonstrated a qualitative association between low WSS and recirculation zones at the carotid bulb where plaques were detected. This is consistent with many reports (e.g. $[10,29-31])$ relating such hemodynamic factors to the localization of atherosclerosis at the carotid bifurcation.

This study indicates that morphology plays an important role on the hemodynamic behavior of the carotid artery bifurcation. It is imperative to include subject-specific morphology and flow velocities in modeling in vivo flow patterns. This novel technology might help to understand the relationship between hemodynamic environment and carotid wall lesions, and have a future impact in carotid stenosis diagnosis and management.

\section{Abbreviations}

3D: Three-dimensional; CCA: Common carotid artery; CFD:

Computational fluid dynamics; DICOM: Digital imaging and communications in medicine; ECA: External carotid artery; ECST: European Carotid Surgery Trial; ICA: Internal carotid artery; WSS: Wall shear stress

\section{Acknowledgments}

This work was partially done in the scope of project PTDC/SAUBEB/102547/2008, "Blood flow simulation in arterial networks towards application at hospital", financially supported by FCT - Fundação para a Ciência e a Tecnologia, Portugal.

\section{Competing interests}

The authors declare no conflict of interest.

References

1. Chiu JJ, Chien S. Effects of disturbed flow on vascular endothelium: pathophysiological basis and clinical perspectives. Physiol Rev 2011; 91(1):327-87.

2. Krams R, Wentzel JJ, Oomen JA, Vinke R, Schuurbiers JC, de Feyter PJ, et al. Evaluation of endothelial shear stress and 3D geometry as factors determining the development of atherosclerosis and remodeling in human coronary arteries in vivo. Combining 3D reconstruc- 
tion from angiography and IVUS (ANGUS) with computational fluid dynamics. Arterioscler Thromb Vasc Biol 1997; 17(10):2061-5.

3. Wang C, Baker BM, Chen CS, Schwartz MA. Endothelial cell sensing of flow direction. Arterioscler Thromb Vasc Biol 2013; 33(9):2130-6.

4. Chandran KB, Vonesh MJ, Roy A, Greenfield S, Kane B, Greene $\mathrm{R}$, et al. Computation of vascular flow dynamics from intravascular ultrasound images. Med Eng Phys 1996; 18(4):295-304.

5. Staub D, Schinkel AF, Coll B, Coli S, van der Steen AF, Reed JD, et al. Contrast-enhanced ultrasound imaging of the vasa vasorum: from early atherosclerosis to the identification of unstable plaques. JACC Cardiovasc Imaging 2010; 3(7):761-71.

6. Swillens A, De Santis G, Degroote J, Lovstakken L, Vierendeels J, Segers P. Accuracy of carotid strain estimates from ultrasonic wall tracking: a study based on multiphysics simulations and in vivo data. IEEE Trans Med Imaging 2012; 31(1):131-9.

7. Antiga L, Piccinelli M, Botti L, Ene-Iordache B, Remuzzi A, Steinman DA. An image-based modeling framework for patient-specific computational hemodynamics. Med Biol Eng Comput 2008; 46(11):1097-112.

8. Botnar R, Rappitsch G, Scheidegger MB, Liepsch D, Perktold K, Boesiger P. Hemodynamics in the carotid artery bifurcation: a comparison between numerical simulations and in vitro MRI measurements. J Biomech 2000; 33(2):137-44.

9. Hoi Y, Wasserman BA, Lakatta EG, Steinman DA. Carotid bifurcation hemodynamics in older adults: effect of measured versus assumed flow waveform. J Biomech Eng 2010; 132(7):071006.

10. Ku DN. Blood Flow in Arteries. Annual Review of Fluid Mechanics 1997; 29(1):399-434.

11. Lee SE, Lee SW, Fischer PF, Bassiouny HS, Loth F. Direct numerical simulation of transitional flow in a stenosed carotid bifurcation. J Biomech 2008; 41(11):2551-61.

12. Zhao SZ, Ariff B, Long Q, Hughes AD, Thom SA, Stanton AV, et al. Inter-individual variations in wall shear stress and mechanical stress distributions at the carotid artery bifurcation of healthy humans. J Biomech 2002; 35(10):1367-77.

13. ECST Collaborators. Randomised trial of endarterectomy for recently symptomatic carotid stenosis: final results of the MRC European Carotid Surgery Trial (ECST). Lancet 1998; 351(9113):1379-87.

14. Gill JD, Ladak HM, Steinman DA, Fenster A. Accuracy and variability assessment of a semiautomatic technique for segmentation of the carotid arteries from three-dimensional ultrasound images. Med Phys 2000; 27(6):1333-42.

15. Santos A, Sousa L, Tavares J, Santos R, Castro P, Azevedo E. Computer simulation of the carotid artery. Cerebrovasc Dis 2012; 33(suppl 1):77.

16. Santos A, Tavares J, Sousa L, Castro C, António C, Santos R, et al. Carotid artery bifurcation modelling from patient $\mathrm{CT}$ angiography and ultrasound technics. Cerebrovasc Dis 2013; 35(suppl 2):47.

17. Santos AMF, dos Santos RM, Castro PMAC, Azevedo E, Sousa L,
Tavares JMRS. A novel automatic algorithm for the segmentation of the lumen of the carotid artery in ultrasound B-mode images. Expert Systems with Applications 2013; 40(16):6570-9.

18. Chan TF, Vese LA. Active contours without edges. IEEE Trans Image Process 2001; 10(2):266-77.

19. Lankton S, Tannenbaum A. Localizing region-based active contours. IEEE Trans Image Process 2008; 17(11):2029-39.

20. Sousa L, Castro C, António C. Blood Flow Simulation and Applications. In: Natal Jorge RM, Tavares JMRS, Pinotti Barbosa M, Slade AP, editors. Technologies for Medical Sciences: Springer Netherlands; 2012. p. 67-86.

21. Sousa LC, Castro CF, Antonio CC, Chaves R. Blood flow simulation and vascular reconstruction. J Biomech 2012; 45(15):2549-55.

22. Lee SW, Steinman DA. On the relative importance of rheology for image-based CFD models of the carotid bifurcation. J Biomech Eng 2007; 129(2):273-8

23. Morbiducci U, Gallo D, Massai D, Ponzini R, Deriu MA, Antiga $\mathrm{L}$, et al. On the importance of blood rheology for bulk flow in hemodynamic models of the carotid bifurcation. J Biomech 2011; 44(13):2427-38.

24. De Santis G, Conti M, Trachet B, De Schryver T, De Beule M, Degroote J, et al. Haemodynamic impact of stent-vessel (mal)apposition following carotid artery stenting: mind the gaps! Comput Methods Biomech Biomed Engin 2013; 16(6):648-59.

25. De Santis G, Mortier P, De Beule M, Segers P, Verdonck P, Verhegghe B. Patient-specific computational fluid dynamics: structured mesh generation from coronary angiography. Med Biol Eng Comput 2010; 48(4):371-80.

26. Kim HJ, Figueroa CA, Hughes TJR, Jansen KE, Taylor CA. Augmented Lagrangian method for constraining the shape of velocity profiles at outlet boundaries for three-dimensional finite element simulations of blood flow. Computer Methods in Applied Mechanics and Engineering 2009; 198(45-46):3551-66.

27. Lee SW, Antiga L, Spence JD, Steinman DA. Geometry of the carotid bifurcation predicts its exposure to disturbed flow. Stroke 2008; 39(8):2341-7.

28. Morbiducci U, Gallo D, Massai D, Consolo F, Ponzini R, Antiga $\mathrm{L}$, et al. Outflow conditions for image-based hemodynamic models of the carotid bifurcation: implications for indicators of abnormal flow. J Biomech Eng 2010; 132(9):091005.

29. Markl M, Wegent F, Zech T, Bauer S, Strecker C, Schumacher M, et al. In vivo wall shear stress distribution in the carotid artery: effect of bifurcation geometry, internal carotid artery stenosis, and recanalization therapy. Circ Cardiovasc Imaging 2010; 3(6):647-55.

30. Meng H, Wang Z, Hoi Y, Gao L, Metaxa E, Swartz DD, et al. Complex hemodynamics at the apex of an arterial bifurcation induces vascular remodeling resembling cerebral aneurysm initiation. Stroke 2007; 38(6):1924-31.

31. Malek AM, Alper SL, Izumo S. Hemodynamic shear stress and its role in atherosclerosis. JAMA 1999; 282(21):2035-42. 